\title{
Capacités d'adaptation des exploitations laitières des hautes terres de la province du Vakinankaratra à Madagascar : impacts de la crise de 2009
}

\author{
Eric Penot ${ }^{1}$ (Gaelle Duba ${ }^{2}$ Paulo Salgado ${ }^{3}$ Patrick Dugué ${ }^{1}$
}

\begin{abstract}
Mots-clés
Bovin laitier, crise économique, évaluation de l'impact, alimentation des animaux, résilience aux crises, Madagascar
\end{abstract}

Accepted: 26 January 2016

Published: 4 July 2016

\begin{abstract}
Résumé
L'objectif de l'étude a été de comprendre les pratiques des exploitants laitiers malgaches après la crise économique (et politique) de 2009, ainsi que d'évaluer son impact sur les revenus des exploitations. L'analyse a été centrée sur les stratégies d'adaptation des éleveurs laitiers pour maintenir le revenu de leurs exploitations face cette période de crise. Elle s'est basée sur des enquêtes réalisées entre 2008 et 2010. Les données ont porté sur trois zones représentant la diversité du triangle laitier des hautes terres, principale région productrice de lait de Madagascar. Une typologie a été réalisée dans un premier temps sur un échantillon de 59 exploitations pour comprendre la rationalité des pratiques d'élevage, en particulier I'alimentation des animaux. Dans un second temps, une modélisation économique avec le logiciel Olympe s'est appuyée sur 21 d'entre elles pour estimer l'impact de la crise sur les revenus des producteurs laitiers. Des scénarios prospectifs rendant compte de l'efficience et des risques des choix techniques par les exploitants ont permis d'explorer l'évolution de la situation avant et après la crise, et de montrer une certaine résilience des exploitations.
\end{abstract}

- Pour citer cet article : Penot E., Duba G., Salgado P., Dugué P., 2016. Adaptability of dairy farms in the highlands of the Vakinankaratra province of Madagascar: Impacts of the 2009 crisis [in French]. Rev. Elev. Med. Vet. Pays Trop., 69 (1): 19-31

\section{INTRODUCTION}

La région des Hautes Terres à Madagascar, située entre 1300 et 2000 mètres d'altitude, est constituée de collines (tanety) et de bas-fonds irrigués qui permettent la culture du riz, souvent prioritaire. L'accroissement démographique et la saturation des terres irriguées impliquent la mise en culture des zones exondées moins fertiles et une augmentation de la production de cultures pluviales dont le riz pluvial (AhmimRichard et al., 2011). Dans ce contexte, une des priorités des projets de développement agricole à partir des années 2000 a été la gestion de la fertilité des terres de tanety et l'accroissement de leur productivité par la vulgarisation des systèmes de culture sur couverture végétale. Pour cela ces projets ont vulgarisé à grande échelle diverses plantes

1. Cirad, UMR Innovation, TA C-85/15, 73 rue Jean-François Breton, 34398 Montpellier Cedex 5, France.

2. Université de Montpelier, Epsed, Montpellier, France.

3. Cirad, UMR Selmet, Antsirabe, Madagascar.

* Auteur pour la correspondance

Tél. : +33(0)467617105

Email : eric.penot@cirad.fr de couverture qui peuvent aussi être valorisées comme plantes fourragères. Progressivement le principal projet de développement régional BVPI-SE/HP ${ }^{4}$ pour qui cette étude a été réalisée - a alors réorienté ses activités vers le renforcement de l'élevage laitier, clé de voute du développement économique régional avec le riz irrigué et le riz pluvial.

Les données collectées et leur traitement proposent, sur la base d'une typologie des élevages laitiers et d'une modélisation des performances des exploitations agricoles laitières de la région des hautes terres, d'évaluer les impacts de la crise économique de 2009 sur l'évolution des pratiques et les revenus des éleveurs. Cette crise économique a fait suite au coup d'état civil de février 2009 aboutissant à une grave crise politique et aux sanctions économiques des bailleurs de fonds ayant pour effet immédiat la disparition de la principale société agro-industrielle d'achat du lait aux éleveurs qui achetait $50 \%$ du lait frais de la région (la société TIKO qui appartenait au président déchu, Ravalomanana). L'analyse a été centrée sur les stratégies d'adaptation des éleveurs laitiers face à cette crise pour maintenir le revenu de leur exploitation dans un contexte d'évolution rapide du marché (baisse de la demande et des prix, puis forte remontée). Des scénarios prospectifs,

4. Projet de développement «Bassin versant, périmètres irrigués, Sud-Est, hauts plateaux », financé par l'Agence française de développement 
rendant compte de l'efficience et des risques des choix techniques qui peuvent être adoptés par les éleveurs, ont permis d'explorer l'évolution de la situation avant et après la crise (depuis 2008). L'objectif de l'article a été de comprendre comment les exploitants laitiers ont réagi à cette crise de 2009, quelles pratiques ont été modifiées, quelles stratégies ont été adoptées et finalement si les exploitations sont sorties renforcées ou affaiblies de cette crise majeure.

\section{Production laitière dans les exploitations agricoles}

La région du Vakinankaratra constitue un bassin de production rizicole, au cœur du «triangle laitier » qui inclut la province du Vakinankaratra (figure 1) et la capitale Antananarivo, et bénéficie de l'implantation ancienne d'industries alimentaires en dehors du secteur laitier (la brasserie STAR, la minoterie Kobama/Moulins de Madagascar, la société exportatrice de légumes Ecofruit, entre autres) qui ont fait de cette région une zone agricole et agro-industrielle (Randrianasolo et al. 2008). Les exploitations agricoles de la région, orientées vers la production de céréales, de fruits, de légumes et de produits animaux, ravitaillent les villes de l'île dont la capitale, notamment en produits laitiers. Plus de $80 \%$ de la production laitière de Madagascar provient de cette région (Rabefenomanantsoa, 2009) ; elle est assurée principalement par un cheptel bovin composé de vaches de race locale, la Rana (croisement entre zébus locaux et divers apports génétiques de races Normande, Pie noire, Bretonne et Gasconne, depuis 1840), et de race améliorée (Pie rouge norvégienne ou PRN), notamment grâce aux actions du centre de recherche appliquée Fifamanor (2008b). La production laitière provient en majorité de petits troupeaux (généralement moins de cinq vaches) intégrés dans des exploitations familiales de polyculture élevage d'une superficie allant de 0,5 à 3 hectares. L'affouragement des bovins est réalisé à partir d'une production fourragère de contre saison (saison sèche) dans les zones irriguées ou les bas-fonds produisant le riz en saison des pluies. Des compléments sont distribués aux animaux ; ils étaient principalement achetés aux collecteurs ou aux coopératives avant 2009, puis produits à la ferme. La totalité des fourrages était distribuée en vert (absence de pratiques de fanage et d'ensilage).

Le contexte de production agricole est celui d'une forte saturation foncière où la sécurité alimentaire reste prioritaire pour la majeure partie des producteurs ayant en moyenne moins d'un hectare de surface cultivable par exploitation, dont une partie seulement est cultivée en contre saison. La diversification laitière implique soit un foncier suffisant pour les cultures fourragères, soit l'utilisation d'une partie des productions vivrières pour la fabrication de la provende des bovins laitiers créant ainsi une situation de compromis / d'adaptation des stratégies paysannes possibles : soit l'agriculteur privilégie

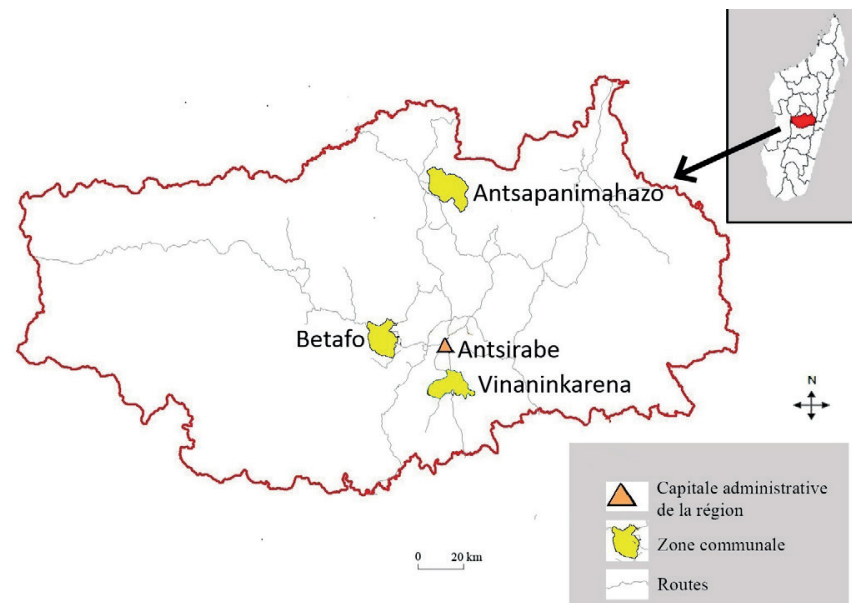

Figure 1 : localisation des trois zones d'étude dans la région du Vakinankaratra (en rouge) à Madagascar. l'autoconsommation et la sécurité alimentaire, soit il privilégie la diversification laitière, la monétarisation de l'exploitation et l'achat de nourriture pour sécuriser les besoins alimentaires de sa famille.

\section{Acteurs de la filière avant la crise}

L'offre de lait a augmenté régulièrement en suivant la demande du marché avec un prix contrôlé par les deux opérateurs principaux (TIKO et Socolait), stable autour de 600 ariary (Ar) par litre $(0,24 €)$ jusqu'en 2008. La a demande augmenté progressivement en particulier sur Antananarivo pour le lait frais, le fromage et les yaourts. La production annuelle du triangle laitier était estimée avant la crise à 110 millions de litres dont seulement 17 millions étaient collectés pour l'exportation hors province. L'organisation de la filière était basée sur les trois principaux circuits. Le premier s'appuyait sur la vente directe et locale de lait frais aux consommateurs locaux et aux magasins de la région ou de la province. Le second concernait les fromageries artisanales locales. Le troisième était le circuit de la vente de lait frais pour la capitale ou vers les grandes industries laitières. Ces trois circuits regroupaient deux types d'acteurs : a) les acteurs traditionnels, qui fournissaient 20 à $30 \%$ du lait cru sur le marché, représentés par les petites exploitations familiales, et b) les acteurs industriels qui fournissaient 70 à $80 \%$ de la production, répartis principalement entre les sociétés TIKO (47\%) et Socolait (14\%) (Fifamanor, 2008a). Ces sociétés ont intégré la filière en fournissant des tanks à lait aux groupements de producteurs, des conseils techniques, comme par exemple les formules de provende, et un système de paiement sécurisé. Fifamanor, projet devenu organisation non-gouvernementale (ONG), fournit en plus du conseil technique des paillettes pour l'insémination artificielle permettant ainsi une amélioration relativement rapide des performances laitières des élevages (accroissement de la base génétique des troupeaux en PRN). Le principal groupe, TIKO, intégrait 4000 producteurs de lait avec 23 centres de collecte, dont 11 réfrigérés (Rabemananbola, 2009). Pendant la pénurie de lait frais, les deux groupes industriels ont importé des quantités massives de lait en poudre pour la production de fromages, de yaourts et de lait concentré. Le lait frais était réservé à la production de lait à ultra haute température.

\section{Crise de 2009, évolution des prix et restructuration de la filière}

La crise politique ${ }^{5}$ et économique de 2009 a eu des répercussions importantes sur la filière lait avec en particulier la fermeture de la Société TIKO et donc l'arrêt immédiat et brutal de la collecte de près de la moitié du lait produit dans la région. Ceci a eu pour effet la chute momentanée du prix d'achat du lait au producteur de 600 à $150 \mathrm{Ar} / \mathrm{L}$ entre février et avril 2009, soit de 0,24 a 0,06€, mettant en danger les marges par litre de lait, la rentabilité globale de l'atelier d'élevage et, indirectement, les investissements effectués par les exploitants dans la production laitière (avec le non-remboursement des crédits) (commun. pers. auprès des opérateurs locaux) (Andriamirija, 2009).

Ainsi, une partie du cheptel a été rapidement vendue entre 2009 et 2010 et les exploitations ont fortement baissé leur production en cherchant à minimiser leurs coûts de production pour l'année 2009. A partir de juillet 2009 la situation s'est stabilisée. Les prix sont revenus au niveau antérieur à la crise $(600 \mathrm{Ar} / \mathrm{L})$ montrant un nouvel équilibre entre l'offre et la demande, sans contrôle du prix par les collecteurs. En 2013, le prix du litre de lait se situait entre 1000 et $1100 \mathrm{Ar} / \mathrm{L}$ $(0,33 € / L$ car la monnaie s'est dévaluée entre temps, passant de

5. Coup d'état civil avec départ du président élu. Cette profonde crise politique a débouché sur des sanctions économiques internationales avec l'arrêt de toutes formes d'aide économique et une profonde récession. Cette crise politique s'est arrêtée avec les élections présidentielles de décembre 2013. 
2500 ariary pour un euro en 2009 à 3000 en 2013) correspondant au maintien d'une forte demande (Penot et Razanakoto, 2012). La filière a poursuivi son effort de restructuration depuis 2009 avec la création de nouveaux débouchés (augmentation très nette de la transformation en fromages et yaourts) et l'apparition de petits collecteurs privés remplaçant la collecte par TIKO, avec cependant moins de moyens (absence de tank à lait réfrigéré pour la collecte locale). Durant cette période, les ONG, coopératives et autres structures d'appui ont été très dynamiques dans la reconstruction de la filière (réseau de collecte, transformation, extension de la production laitière sur Antananarivo et production de provende locale). La production de lait du triangle laitier en 2012 a été estimée à $80 \%$ de celle de 2008 (Penot et al., 2012), avec une certaine atomisation des collecteurs et une montée en puissance de la seconde principale compagnie privée restant sur le marché (Socolait), devenue la première après 2009. Les exploitations ont modifié leurs pratiques et leurs coûts de production pour s'adapter à ces nouvelles conditions de marché.

\section{Pratiques d'alimentation du cheptel laitier avant la crise}

La majorité des exploitants régulaient l'alimentation des vaches laitières en fonction des saisons sur la base d'aliments frais. Durant la saison des pluies (novembre à avril) le bétail (vaches laitières et zébus de trait) était alimenté exclusivement sur pâturage naturel ou cultivé, qui présentait des plantes fourragères comme Aristida rufescens, maïs fourrager, Pennisetum cv. Kizozi, Brachiaria (ruziziensis, decumbens ou brizantha) et Chloris gayana. En saison sèche (maioctobre), la production de biomasse était limitée en raison des températures basses (saison hivernale). A cette période les vaches laitières étaient essentiellement alimentées avec les résidus de culture (pailles de riz et d'orge) et avec les graminées des zones tempérées cultivées (avoine) sur tanety (cultures sur collines pluviales par opposition aux cultures irriguées sur rizières avec maîtrise de l'eau), et en contresaison dans les rizières (avoine, ray-grass ; Fifamanor et al., 2008). Les techniques de conservation de fourrages (par exemple foin, ensilage) et les méthodes de valorisation des pailles (traitement de la paille de riz à l'urée) étaient presque inexistantes chez les éleveurs. Comme compléments, les éleveurs utilisaient les drèches de brasserie toute l'année dans la région d'Antsirabe et de Betafo en raison de la proximité avec la brasserie STAR. L'utilisation de concentrés était très variable en quantité et qualité d'une exploitation à l'autre : entre 72000 et 3 millions d'ariary (30-1200€) par exploitation par an, avec une à six vaches selon les exploitations. Elle dépendait des revenus, de la disponibilité économique et du niveau de formation des exploitants (encadrés ou non par les organismes d'appui ou les coopératives). Chez tous les éleveurs, les fumures organiques étaient utilisées pour la fertilisation des rizières irriguées et des parcelles vivrières sur zones exondées (tanety), particulièrement pour les cultures à forte valeur ajoutée (pomme de terre, riz pluvial). Suite aux activités de formation et de vulgarisation de Fifamanor, les modes d'alimentation du troupeau laitier sont basés depuis le début des années 2000 sur l'utilisation des aliments suivants :

- l'herbe en saison des pluies ; les cultures fourragères sur tanety (Kizozi, Relaza, Brachiaria, Setaria, Desmodium) ; les cultures fourragères sur rizières (avoine, ray-grass) ; les herbes ramassées (herbes sauvages) ; et les résidus de culture (pailles de riz, d'orge ou de maiis) ;

- différents types de concentrés (Kasprzyk et al., 2008) ; des provendes fermières à faible valeur alimentaire (son de riz, excédents vivriers comme la patate douce, pomme de terre, manioc en vrac) ; des provendes fermières à forte valeur alimentaire (maïs broyé, son de riz, soja, manioc, son de riz) ; et des provendes fermières ou commerciales complètes à bonne valeur alimentaire (fabrication selon la formule, à partir de tourteau, de maïs, de soja, de son de riz, de coquillages, ou autre).

\section{MATERIEL ET METHODES}

\section{Trois zones d'étude}

Nous avons retenu trois zones différentes du point de vue de la filière laitière (production -commercialisation) (tableau I). Le village de Vinaninkarena, situé sur une route nationale, est représentatif de la zone proche de la ville d'Antsirabe (à $10 \mathrm{~km}$ ) et de la bonne accessibilité à son marché. La région de Betafo représente la zone la plus riche (terres volcaniques fertiles, disponibilité toute l'année en eau pour l'irrigation ou autre usage), proche d'Antsirabe (environ $20 \mathrm{~km}$ ) et avec la possibilité de production fourragère en saison sèche. La région d'Antsapanimahazo Faratsiho a été choisie pour représenter la situation des zones enclavées (72 km au nord-ouest d'Antsirabe), à une altitude de $1700 \mathrm{~m}$ (figure 1 ; tableau I).

\section{Enquêtes et modélisation}

L'étude a été centrée sur les stratégies d'adaptation des éleveurs laitiers pour maintenir le revenu de l'exploitation dans un contexte de fortes turbulences politiques et économiques, et basée sur des données d'enquêtes. Trois enquêtes ont été réalisées entre 2008 et 2010. Les deux premières ont permis d'acquérir des informations sur les structures des exploitations laitières et les modes d'alimentation des animaux.

La première enquête a été réalisée sur 30 exploitations dans la zone de Betafo en 2008 (Kasprzyk et al., 2008) et sur 29 exploitations dans les zones de Vinaninkarena et d'Antsapanimahazo en 2009 (Rarivoarimanana, 2010), soit 59 exploitations au total. Cette enquête a débouché, entre autres, sur une typologie des exploitations laitières et une typologie des modes d'alimentation.

La seconde enquête a été menée en 2010 pour caractériser plus en détail 21 exploitations laitières issues de la première enquête (Duba, 2010) afin de modéliser des fermes représentatives de chaque type à l'aide d'Olympe (créé par Attonaty, INRA, 2000), logiciel de simulation technico-économique du fonctionnement de l'exploitation agricole et du ménage associé au sein d'un système d'activité (Penot, 2012). Les 21 exploitations enquêtées ont permis de construire les scénarios présentés ci-dessous. Les résultats ont été validés lors de séances de restitution de groupe aux paysans des trois zones et avec l'équipe du projet BVPI-SE/HP.

La troisième enquête, appelée « réaction face à la crise », a été conduite sur 45 exploitations en 2010 (soit un an après la crise majeure de février 2009), 15 exploitations dans chacune des trois zones parmi l'échantillon initial de 59 exploitations enquêtées en 2008-2010, afin d'identifier les changements de pratiques et de préciser des itinéraires techniques pour chaque type d'exploitation, tant pour les systèmes de culture que pour les systèmes d'élevage qui ont ensuite été utilisés dans la modélisation. L'objectif de cette dernière enquête a été de comprendre la perception de la crise par les exploitants, et les raisons et les motivations qui ont encouragé les exploitants à prendre certaines décisions tactiques (modes d'alimentation) et stratégiques (vente, puis rachat d'animaux). Des scénarios ont été faits en reconstruisant pas à pas les années 2008 à 2010, pour identifier l'évolution des coûts et des sources de revenus et identifier l'impact économique des pratiques en évolution.

Nous disposions ainsi de deux bases de données : « exploitation/ données générales et systèmes d'alimentation » dans Excel pour 89 exploitations, et « exploitations complètes modélisées » dans Olympe pour 21 exploitations.

L'utilisation d'Olympe (Penot, 2012) pour cette étude a été conditionnée par plusieurs critères : la grande facilité d'utilisation du logiciel, son adaptation à la question posée, et la possibilité de simuler différents scénarios permettant de quantifier le risque et les indicateurs 


\section{Tableau I}

Comparaison des caractéristiques des différentes zones d'étude sur les hautes terres à Madagsacar

Antsapanimahazo

\section{Caractéristiques biophysiques}

Accès difficile au marché, zone enclavée, 72 km d'Antsirabe

Bonne fertilité, sols volcaniques calcaire et argileux-quartzique

Altitude $1700 \mathrm{~m}$, température moyenne à basse en hiver, risque de gel

Pluviométrie importante (1900 mm/an)

\section{Vinaninkarena}

Bon accès au marché, 10 km d'Antsirabe par la RN7

Fertilité moyenne, sol ferralitique/ fluviolacustre, difficultés de maîtrise de l'eau pour l'irrigation des rizières

Altitude 1500 m, température moyenne (zéro en juin)

Pluviométrie moyenne (1100 mm/an)

\section{Betafo}

Assez bon accès au marché, 22 km d'Antsirabe

Bonne fertilité, sols bruns volcaniques

Altitude 1500 m, température moyenne

Pluviométrie bonne (entre 1000 et $1500 \mathrm{~mm} / \mathrm{an})$

\section{Caractéristiques des exploitations agricoles}

Productions agricoles principales : pomme de terre, haricot, patate douce, maïs, riz, manioc

Pression foncière faible

Revenus essentiellement de la production agricole (peu de hors exploitation)
Productions agricoles principales : riz, pomme de terre, manioc, maïs, cultures maraîchères (tomate, ail)

Pression foncière forte

Revenus hors exploitation importants au sein de l'exploitation
Productions agricoles principales : riz, maïs, manioc, pomme de terre, haricot les cultures de rente tiennent une place importante

Pression foncière forte

Revenus hors exploitation importants au sein de l'exploitation

\section{Caractéristiques de l'élevage laitier}

Nb. exploitations laitières : 617

$\mathrm{Nb}$. vaches laitières : 1053

La majorité des exploitations ne possède qu'une à deux vaches laitières en production

Potentiel génétique faible

Production laitière par vache faible

Production totale de la zone : $650000 \mathrm{~L} / \mathrm{an}$

Débouchés de vente faibles (épicerie ou fromagerie)

Prix du lait 2010 dans la zone : 450-500 Ar/L

Reproduction des vaches laitières : monte naturelle
Nb. exploitations laitières : 74

$\mathrm{Nb}$. vaches laitières : 186

$43 \%$ des exploitations possèdent une vache en production et $44 \%$ en possèdent deux ou trois

Potentiel génétique moyen

Production laitière par vache moyenne

Production totale de la zone : 217000 L/an

Débouchés de vente importants (Antsirabe)

Prix du lait 2010 dans la zone : $600 \mathrm{Ar} / \mathrm{L}$ monte naturelle ; $22 \%$ insémination artificielle
Reproduction des vaches laitières : $78 \%$

Nb. exploitations laitières : 478

$\mathrm{Nb}$. vaches laitières : 1793

La majorité des exploitations possède plus de trois vaches laitières en production

Potentiel génétique bon

Production laitière par vache bonne

Production totale de la zone :

2860000 L/an

Débouchés de vente importants (marché local ou Antsirabe)

Prix du lait 2010 dans la zone : $600 \mathrm{Ar} / \mathrm{L}$

Reproduction des vaches laitières : insémination artificielle en majorité

CSA lavoko Betafo, 2010

Données d'enquêtes, Rarivoarimanana 2009-2010

Données Cirdr Betafo 2007-2008 et Fifamanor 2009 majeurs des stratégies paysannes. Les informations constituant la base de données ont été séparées en trois modules (atelier d'élevage ou de productions végétales, entreprise, et famille / compte privé).

La structure du logiciel Olympe permet d'étudier les résultats économiques des systèmes de culture et d'élevage, puis celui du système de production à travers le compte d'exploitation général (CEG). Cependant, le concept de système de production n'a pas suffit à décrire les exploitations familiales agricoles. Pour les exploitations agricoles malgaches, l'unité de production familiale s'est superposée à l'unité de consommation et à celle d'accumulation. Il existait donc des interactions très fortes entre l'exploitation agricole et le ménage, qui nous ont amenés à prendre en compte l'ensemble des décisions de production et de consommation de la famille (incluant le choix des cultures mais aussi les activités extra-agricoles et la part de la production 
autoconsommée). Ceci nous a conduits à étudier le revenu total de l'exploitation, composé des revenus agricoles et des revenus non-agricoles. Le revenu net agricole est issu de la somme des marges nettes par système de culture et d'élevage dans l'exploitation. En l'absence de crédit (depuis 2009) et donc de charges financières, la marge brute est égale à la marge nette. Le revenu net agricole est donc égal au résultat issu du CEG, équivalent à la somme des marges brutes par activité, moins les charges fixes de l'exploitation (très faibles voire inexistantes dans la plupart des cas dans le contexte malgache de la petite agriculture familiale).

Olympe est un logiciel de type tableau avec une interface spécifique, des tableaux de sortie automatisés, personnalisables à volonté (par exemple variables calculées, nouveaux tableaux, construction des aléas). L'analyse est faite pas à pas : il n'y a pas de module d'optimisation (type solveur) ni de module de programmation linéaire. Le logiciel est adapté par sa robustesse et sa simplicité à une analyse de gestion sur les coûts et revenus de l'exploitation agricole et du ménage associé (système d'activité).

\section{Typologie d'exploitation}

La typologie des exploitations laitières a été obtenue sur la base de deux critères distincts (enquête $n^{\circ} 1$ ) : a) le niveau de diversification des revenus dans l'exploitation (revenus d'origine agricoles et nonagricoles ou hors exploitation) et b) la nature du système d'alimentation combinant la surface fourragère disponible par tête de bovin laitier (SFDB 6) et l'accès aux concentrés et aux drèches de brasserie. L'origine des revenus du ménage était importante car une partie des revenus issus des activités non-agricoles était souvent réinvestie dans l'élevage.

Trois catégories de provende (A, B, C) ont été considérées en fonction de leur valeur nutritive en énergie et en protéines. Par ailleurs, les éleveurs de type $\mathrm{S} 1$ utilisaient très peu de fourrages cultivés et basaient l'alimentation sur les ressources naturelles ; ils se différenciaient des autres types qui utilisaient des concentrés et des pâturages cultivés. Il s'agissait de systèmes très peu intensifiés demandant un faible investissement monétaire (pas ou peu de concentrés). Généralement, ces exploitants élevaient seulement une vache laitière. Les systèmes intermédiaires de type $\mathrm{S} 2$ valorisaient de façon importante les résidus de récolte issus des cultures de l'exploitation. Il s'agissait de systèmes peu intensifiés en intrants mais dont les besoins en surface vivrière et en travail étaient importants (stocks importants de résidus

${ }^{6}$ La SFDB par exploitation a été déterminée selon la formule suivante : surface fourragère sur tanety + surface fourragère sur rizière / (nombre de vaches laitières $+[\text { (nombre de génisses }+ \text { nombre de veaux et velles }+ \text { nombre de taurillons })^{*} 0,25$ + nombre de taureaux ; indicateur synthétique « surface » utilisé par Fifamanor. de culture). Les systèmes de type $\mathrm{S} 3$ intégraient des pâturages cultivés (incluant les surfaces fourragères de tous types). Les systèmes de type S4 étaient les plus intensifs en capital ou en intrants, avec des vaches laitières tenues en stabulation permanente et l'utilisation de provende permettant une production de lait plus importante (tableau II).

\section{RESULTATS ET DISCUSSION}

\section{Evolutions des systèmes d'alimentation suite à la crise de 2009}

Si en 2008 de nombreux éleveurs utilisaient les provendes vendues par TIKO ou les coopératives, en 2012 la grande majorité des exploitants fabriquaient eux-mêmes leurs provendes fermières ou ont trouvé de nouveaux distributeurs (coopératives locales ou vendeurs privés) de qualité hétérogène et de valeur nutritive très différente.

\section{Antsapanimahazo}

Cette zone était caractérisée majoritairement par des exploitants (19 enquêtés et 7 modélisés) du type S1 (alimentation sur ressources naturelles) qui possédaient peu de surface fourragère cultivée et avaient la possibilité d'utiliser des ressources naturelles au pâturage (figure 2). Une seule exploitation sur 19 de type S3 (association d'une part importante de fourrages cultivés et de pâturage) a été trouvée. Enfin, $20 \%$ des exploitants de cette zone, encadrés par des techniciens, ont mis en place des cultures fourragères diversifiées leur permettant d'alimenter leurs vaches laitières de façon plus intensive (S4).

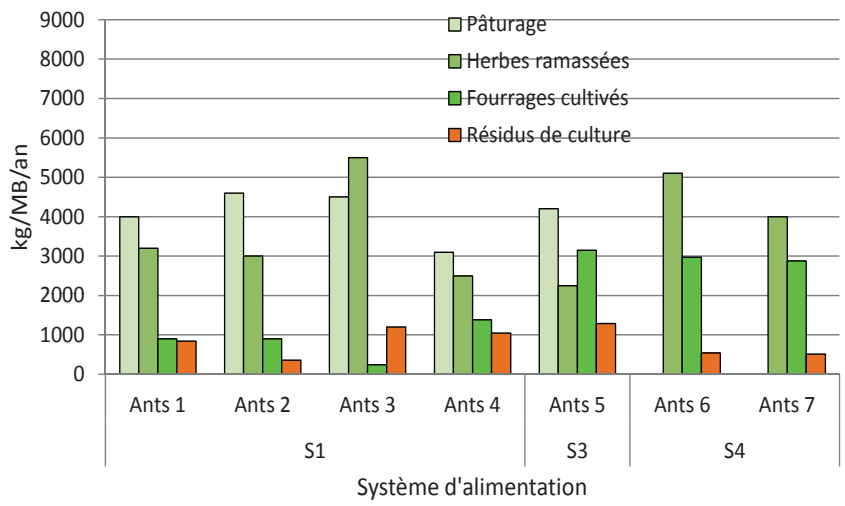

Figure 2 : ration fourragère annuelle distribuée par les exploitants d'Antsapanimahazo dans la province du Vakinankaratra à Madagascar en 2010 ; MB : matière brute.

\section{Tableau II}

Typologie d'exploitation basée sur les systèmes d'alimentation (S1-S4) des vaches laitières sur les hautes terres à Madagsacar

\begin{tabular}{|c|c|c|c|c|c|c|c|}
\hline \multirow[t]{2}{*}{ Type } & \multicolumn{4}{|c|}{$\begin{array}{l}\text { Base du système d'alimentation distribué } \\
\text { (part de la ration annuelle) }\end{array}$} & \multicolumn{3}{|c|}{$\begin{array}{l}\text { Valeur nutritive } \\
\text { du complément et type }\end{array}$} \\
\hline & FC & Conduite & HR & $\mathbf{R C}$ & Faible & Moyen & Complet \\
\hline S1 & \multirow[t]{2}{*}{ Peu de $\mathrm{FC}<20 \%$ de la ration } & Pâturage (P) & $\mathrm{HR}+\mathrm{P}>50 \%$ & $\mathrm{RC}<20 \%$ & S1 A & S1 B & \\
\hline S2 & & Stabulation & $\mathrm{HR}>50 \%$ & $\mathrm{RC}>20 \%$ & S2 A & S2 B & \\
\hline S3 & \multirow[t]{2}{*}{ FC $>20 \%$ de la ration } & Pâturage & $\mathrm{HR}+\mathrm{P}<50 \%$ & $\mathrm{RC}<20 \%$ & & S3 B & \\
\hline S4 & & Stabulation & $\mathrm{HR}<50 \%$ & $\mathrm{RC}<20 \%$ & & S4 B & S4 C \\
\hline
\end{tabular}

FC : fourrages cultivés ; HR : herbes ramassées ; RC : résidus de culture 


\section{Vinaninkarena}

Cette zone était caractérisée majoritairement par des exploitants ( $\mathrm{n}=$ 20) du type $S 2$ (peu de fourrages cultivés et stabulation) qui, soumis à une pression foncière importante, étaient en majorité contraints de maintenir les vaches en stabulation. Les résidus de culture (paille de riz) étaient très importants dans la ration fourragère majoritairement de type $\mathrm{S} 2$ (figure 3). Des exploitants de type $\mathrm{S} 1$ étaient également représentés ; il s'agissait en particulier d'exploitants dont l'activité principale n'était pas l'élevage. Les exploitants possédaient souvent des revenus non-agricoles importants leurs permettant de ne pas investir dans l'alimentation animale et de se contenter de faibles productions laitières.

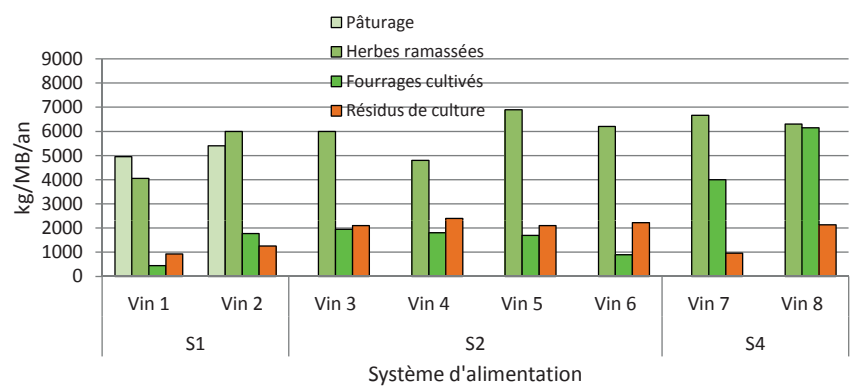

Figure 3 : ration fourragère annuelle distribuée par les exploitants de Vinaninkarena dans la province du Vakinankaratra à Madagascar en 2010 ; MB : matière brute.

\section{Betafo}

Cette zone était caractérisée majoritairement par des exploitants ( $\mathrm{n}=$ 20) du type $S 4$ (fourrages cultivés et stabulation), spécialisés dans l'activité laitière. Ils mettaient en place des cultures fourragères pour alimenter correctement les animaux et se rapprocher de l'optimum économique de production laitière pour le producteur (figure 4). Il s'agissait de systèmes plus intensifiés dont l'investissement dans l'életation. En parallèle, se trouvaient des exploitants éloignés de la route nationale et du marché, qui alimentaient leur bétail par le pâturage sur parcours naturels (type S1).

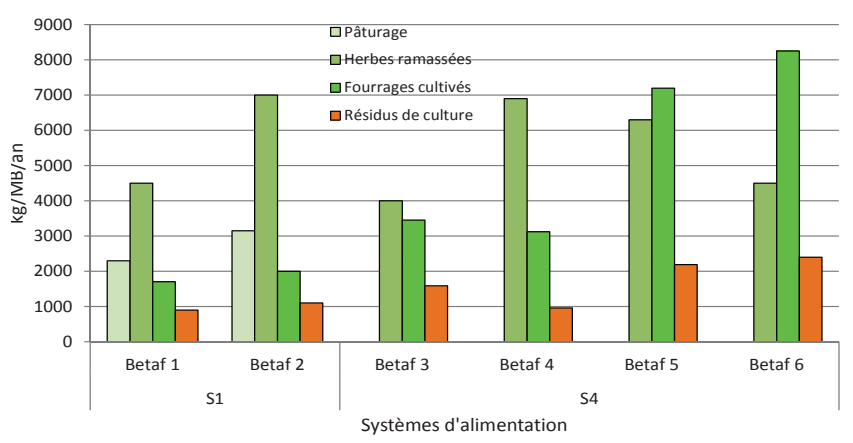

Figure 4 : ration fourragère annuelle distribuée par les exploitants de Betafo dans la province du Vakinankaratra à Madagascar en 2010 ; MB : matière brute.

\section{Systèmes d'alimentation et marge brute des exploitants}

Ces résultats (figure 5) proviennent de l'enquête $n^{\circ} 2$. Les exploitations agricoles alimentant les vaches laitières de façon extensive avec peu d'intrants alimentaires (S1 A et S1 B) avaient une marge nette annuelle faible comprise entre 800000 et 2200000 Ar (respectivement 295 et $815 €$ ) ; pour comparaison, le salaire minimum légal est de 720 k.Ar/an $(266 €)$. Les exploitants valorisant essentiellement les résidus de récolte pour l'alimentation des vaches (S2 A et S2 B) possédaient une marge légèrement supérieure avec une moyenne de $3500000 \mathrm{Ar} / \mathrm{an}(1290 €)$. Ceci n'était pas directement lié à l'atelier laitier, mais plutôt à la surface plus importante de cultures en rizière qui leur permettait de distribuer plus de paille de riz en saison sèche. Les revenus agricoles de l'unique exploitant enquêté en système de type S3 étaient élevés (1,7 million Ar) en raison de la grande superficie de terres possédées lui permettant un pâturage important, notamment après la récolte sur les résidus de culture. Enfin les exploitations agricoles de type $\mathrm{S} 4$ possédaient des marges nettes plus importantes que celles de types $\mathrm{S} 1$ et $\mathrm{S} 2$. Toutefois on remarque que trois exploitants (sur 20) possédaient une marge et un revenu d'exploitation supérieurs à la moyenne. Il s'agissait de grosses exploitations (possédant plus de cinq têtes) situées à Betafo, dont l'investissement dans l'élevage laitier était important, l'un d'eux ayant mis en place une fromagerie.

L'analyse a été faite à partir de la marge brute d'exploitation pour mesurer l'impact global, au niveau du revenu de l'exploitation, des changements techniques. Pour les types S1 et S2 l'impact du changement de type d'alimentation suite à la crise a fortement diminué les différences entre exploitations avec des comportements identiques (réduction de la ration et de la production et vente d'une partie des animaux), alors que pour le type S4 les différences ont montré, d'une part, des accès à la ressource foncière très divers et, d'autre part, des stratégies paysannes très différenciées, aboutissant à une gamme de marge brute (revenu brut agricole) plus large.

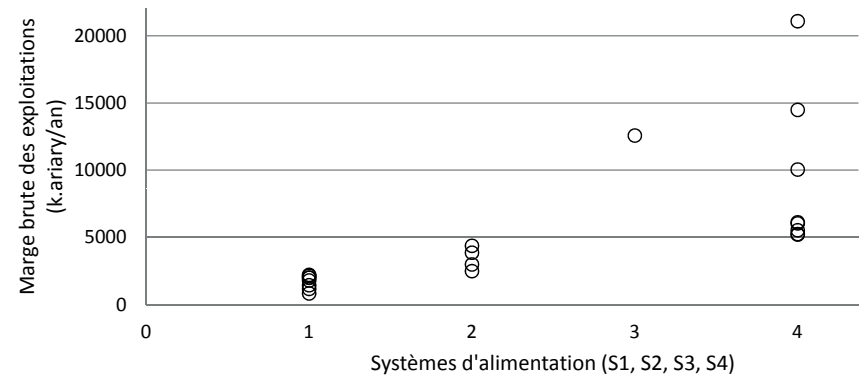

Figure 5 : marge brute des exploitants enquêtés en 2010 dans la province du Vakinankaratra à Madagascar selon le système d'alimentation des vaches laitières (S1 à S4). En l'absence de frais financiers et de frais fixes : marge brute annuelle = marge nette.

\section{Place de l'élevage laitier au sein de l'exploitation agricole}

Dans la région d'Antsapanimahazo, le revenu des exploitations était principalement issu des cultures vivrières (figure 6) en partie vendues à l'extérieur et en partie autoconsommées. L'élevage laitier ne représentait qu'un complément dans les revenus de l'exploitation, de l'ordre de $10 \%$ du total. La zone de Vinaninkarena était très marquée par la présence de revenus non-agricoles (hors exploitation) du fait de la proximité de la ville d'Antsirabe. Il en était de même à Betafo, bien que pour cette dernière, la plupart des revenus non-agricoles provenaient de pensions des producteurs dont l'activité principale n'était pas agricole avant leur retraite. L'élevage de vaches laitières avait une place importante à Vinaninkarena (20\% des revenus). Cependant, c'était à Betafo que l'élevage laitier contribuait de façon la plus significative aux revenus de l'exploitation (34\% des revenus). Il s'agissait d'éleveurs spécialisés dans la production laitière. La plupart des autres produits agricoles n'étaient pas vendus car ils étaient autoconsommés.

La plus grande part des charges de l'élevage provenait du complément alimentaire (tableau III), et les différences dans les dépenses liées à 


\section{Antsapanimahazo}

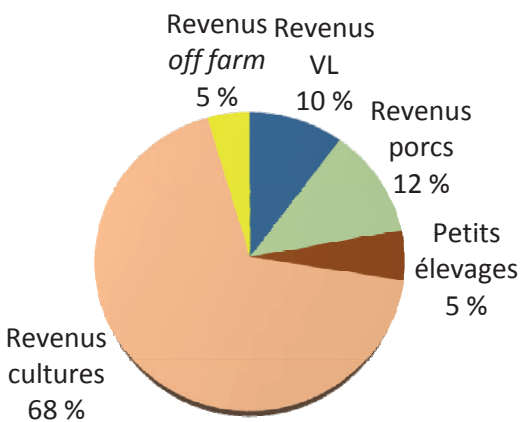

Vinaninkarena

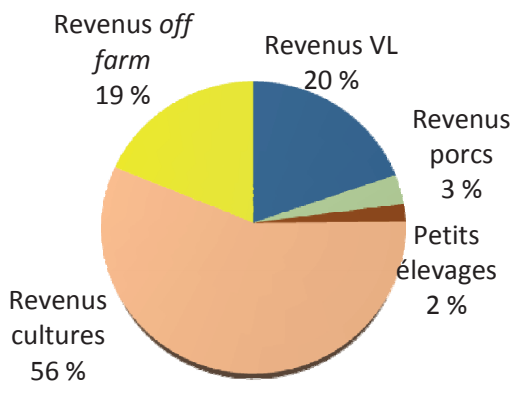

Betafo

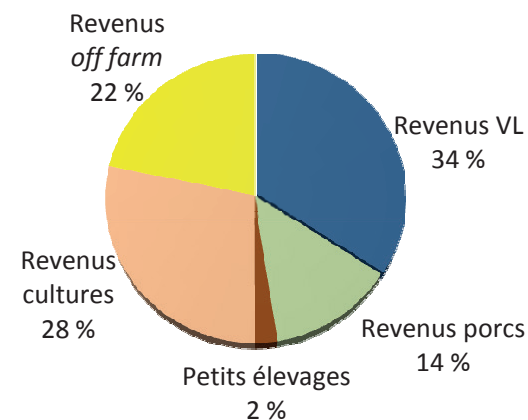

Figure 6 : composition des revenus des exploitants laitiers au sein des différentes zones d'étude dans la province du Vakinankaratra à Madagascar en $2010 ;$ VL : vache laitière.

\section{Tableau III}

Coûts de production et marges brutes issus de l'élevage laitier sur les hautes terres à Madagsacar pour I'année 2010

\begin{tabular}{|c|c|c|c|c|c|c|c|c|c|}
\hline \multirow[t]{2}{*}{ Expl } & \multirow[t]{2}{*}{ Sys } & \multirow[t]{2}{*}{ TC } & \multicolumn{3}{|c|}{ Dépenses (ariary/an) } & \multirow{2}{*}{$\begin{array}{c}\text { Dépense } \\
\text { totale } \\
\text { (Ar/an) }\end{array}$} & \multirow{2}{*}{$\begin{array}{l}\text { Prod lait } \\
\text { (L/an) }\end{array}$} & \multirow{2}{*}{$\begin{array}{c}\text { Marge } \\
\text { brute } \\
\text { (Ar/an) }\end{array}$} & \multirow{2}{*}{$\begin{array}{c}\text { Coût } \\
\text { prod } \\
1 \mathrm{~L}(\mathrm{Ar})\end{array}$} \\
\hline & & & $\begin{array}{l}\text { Cultures } \\
\text { fourragères }\end{array}$ & $\begin{array}{l}\text { Complément } \\
\text { alimentaire }\end{array}$ & $\begin{array}{c}\text { Soins } \\
\text { vétérinaires }\end{array}$ & & & & \\
\hline Ants 3 & S1 & A & 183750 & 265500 & 17400 & 466650 & 2160 & 88350 & 216 \\
\hline Ants 4 & S1 & B & 167800 & 376500 & 13200 & 557500 & 1434 & 132500 & 389 \\
\hline Vin 3 & $\mathrm{~S} 2$ & A & 205420 & 105000 & 6600 & 317020 & 1680 & 450980 & 189 \\
\hline Vin 2 & $\mathrm{~S} 2$ & $\mathrm{~B}$ & 177500 & 227250 & 17200 & 421950 & 1900 & 718050 & 222 \\
\hline Ants 5 & S3 & B & 195500 & 240000 & 27120 & 462620 & 1468 & -136420 & 315 \\
\hline Bet 3 & S4 & $\mathrm{C}$ & 782100 & 1146000 & 43200 & 1971300 & 4305 & 566700 & 458 \\
\hline Bet 5 & S4 & $\mathrm{C}$ & 2006000 & 2406000 & 47600 & 4459600 & 13304 & 1360400 & 335 \\
\hline
\end{tabular}

Expl : exploitant ; Sys : système d'alimentation ; TC : type de complément ; Prod lait : production laitière ; Coût prod $1 \mathrm{~L}$ : coût de production d'un litre de lait Ants : Antsapanimahazo; Vin : Vinaninkarena ; Bet : Betafo

l'élevage étaient fonction des systèmes d'alimentation utilisés et donc du type d'exploitation. En particulier, après la crise de 2009 la production de provende par les éleveurs a représenté une stratégie prioritaire pour limiter les coûts de production, et assurer une alimentation de qualité et la rentabilité de l'atelier lait. Ainsi, la production laitière annuelle a dépendu directement de la qualité et de la quantité de la provende distribuée.

Le coût de production d'un litre de lait (vendu en moyenne autour de $600 \mathrm{Ar} / \mathrm{L}$ ) a été faible dans les systèmes associant le pâturage avec un apport faible en aliments concentrés (Ants 3 et 5 ; Vin 3 et 2 ; tableau III) et une marge brute de l'élevage basse du fait d'une quantité de lait produit relativement faible en 2010 (année charnière dans la crise). Pour les systèmes d'alimentation associant le pâturage et un concentré à valeur nutritive moyenne (type B), le coût de production du lait a été plus important (Ants 4) à cause du coût élevé de la provende. Les exploitations pouvant distribuer de la paille de riz de manière importante au cours de la saison sèche (type S2) et qui distribuaient également une provende de qualité nutritive moyenne ont eu un faible coût de production et une marge brute intéressante en raison d'une production laitière moyenne et d'une vente de lait à $600 \mathrm{Ar} / \mathrm{L}$. Dans les systèmes intensifiés (S4), le coût de production du litre de lait élevé a été compensé par une production de lait maximisée.

\section{Conséquences de l'arrêt de la société TIKO}

La fermeture de TIKO et donc l'arrêt de l'approvisionnement en provende des éleveurs en contrat avec cette société ont contraint ces derniers à fabriquer leur propre provende, conseillés pour cela par les coopératives et Fifamanor qui ont rapidement mis à disposition des formules de provende basées sur des ressources locales (Carimentrand, 2011).

\section{Stratégies de réaction des producteurs}

Les scénarios modélisés par Olympe représentaient une simulation des pratiques des 21 exploitants illustrant leurs réactions face au rétrécissement du marché, à l'évolution des prix du lait et des intrants, et à la nécessité de fabriquer à la ferme la provende. Ces scénarios ont été reconstitués sur la base des dires d'acteurs sur les trois années de l'étude (2008-2010) et ont permis de visualiser et de comparer les évolutions. L'analyse de ces scénarios a permis de décrire les quatre grandes stratégies adoptées par les éleveurs (figure 7). Puis des scénarios complémentaires prospectifs ont été réalisés pour tester différentes formules de provende et de rationnement des animaux par rapport aux objectifs de production choisis ou possibles afin d'identifier les meilleures provendes potentielles (le résultat de ces scénarios prospectifs n'est pas présenté dans cet article).

Concernant l'alimentation des vaches laitières, les éleveurs ont préféré arrêter l'achat de concentrés alimentaires dès février 2009 afin de limiter les charges opérationnelles, ce qui les a conduit aussi à limiter la production laitière pour s'accorder avec une demande réduite de $50 \%$ en quelques semaines (février-mai 2009). Par ailleurs, les concentrés sont devenus introuvables en quelques mois. Ils ont alors choisi de mieux valoriser les résidus de culture ainsi que les 


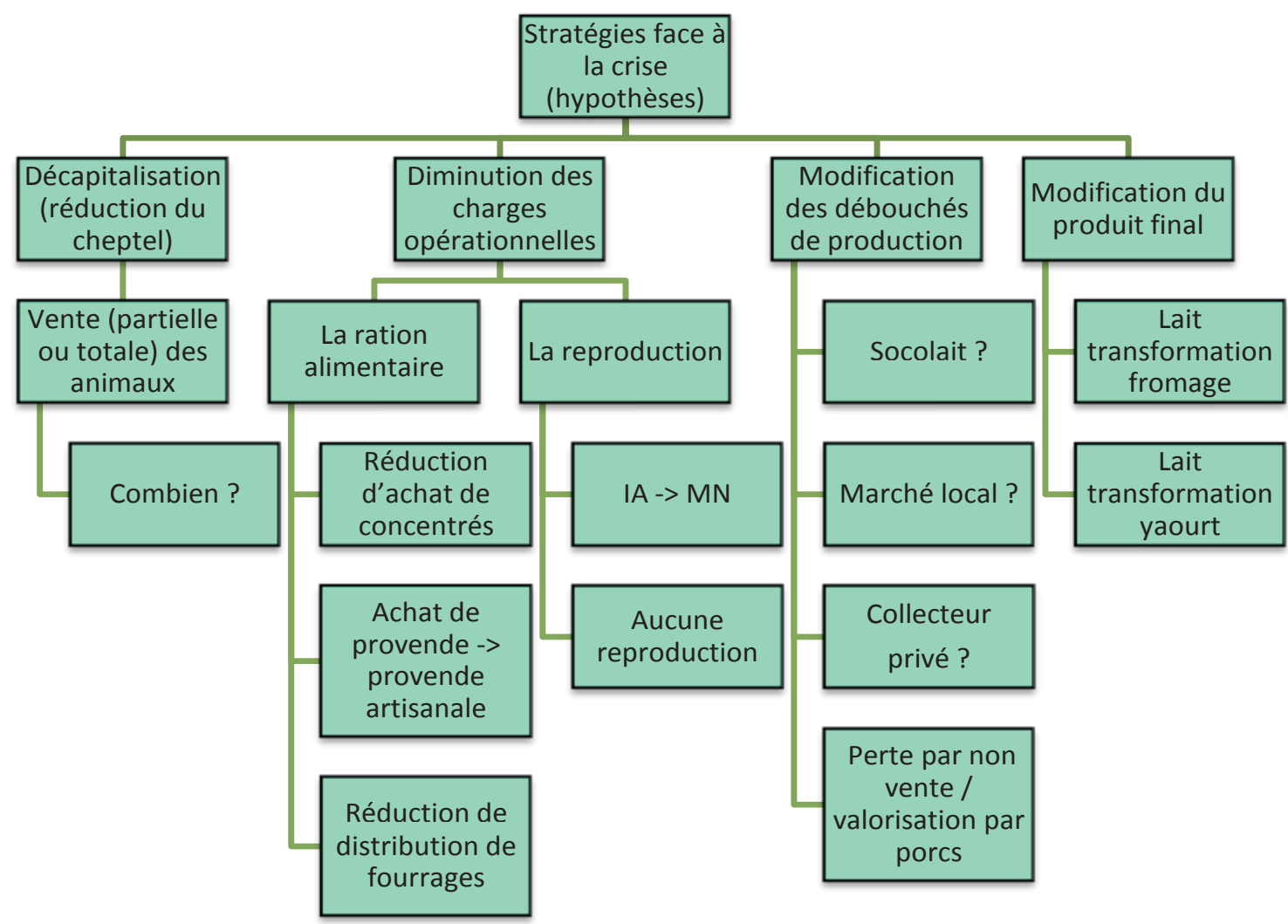

Figure 7 : stratégies possibles mises en place par les éleveurs laitiers de la province du Vakinankaratra à Madagascar après la crise de 2009. $I A$ : insémination artificielle; $M N$ : monte naturelle.

herbes ramassées pour nourrir leurs troupeaux et réduire la production laitière pour la période 2009-2010.

La décapitalisation par vente d'animaux a été effective pour la majeure partie des éleveurs (passage de trois à deux vaches laitières par exemple) avec rachat d'animaux à partir de 2011-2012 quand le prix du lait est passé à $1000 \mathrm{Ar} / \mathrm{L}$. Parmi les éleveurs laitiers possédant plusieurs vaches laitières, $80 \%$ à Betafo et $40 \%$ à Vinaninkarena en ont ainsi vendu pour diminuer leur production laitière (tableau IV). Les autres éleveurs ont limité la production des vaches laitières sans vendre ces dernières mais en diminuant la distribution de concentré ration (87\% à Antsapanimahazo, $60 \%$ à Vinaninkarena et $20 \%$ à Betafo). En cas de vente de vaches, l'argent obtenu a été réinvesti dans l'achat de terres cultivables ou dans l'élevage porcin qui a un retour sur investissement plus rapide et un investissement initial moindre. Les revenus de l'élevage porcin ont permis à l'exploitation de compenser partiellement les pertes sur l'atelier lait ou la baisse du

\section{Tableau IV}

Vente des vaches laitières sur les hautes terres à Madagascar entre 2009 et 2010

\begin{tabular}{lcccc} 
& $\begin{array}{c}\text { Eleveurs }{ }^{\mathbf{1}} \\
(\%)\end{array}$ & Vaches $^{\mathbf{2}}$ & $\begin{array}{c}\text { Eleveurs } \\
\text { enquêtés } \\
\text { (nb.) }\end{array}$ & $\begin{array}{c}\text { Vaches/ } \\
\text { exploitation } \\
\text { (nb. moyen) }\end{array}$ \\
\hline Ants & 13 & 1,0 & 15 & 1 à 2 \\
Vin & 40 & 1,3 & 15 & 2 à 3 \\
Bet & 80 & 2,6 & 15 & $>3$
\end{tabular}

${ }^{1}$ Eleveurs déclarant avoir vendu au moins une vache laitière entre 2009 et 2010 ${ }^{2} \mathrm{Nb}$. moyen de vaches déclarées avoir été vendues par les exploitations ayant vendu des vaches

Ants : Antsapanimahazo ; Vin : Vinaninkarena ; Bet : Betafo revenu par rapport à avant 2009, au risque de pertes importantes en cas de peste porcine africaine (prévalence de $20 \%$ ).

L'objectif de réduction des charges opérationnelles a aussi abouti à l'abandon de l'insémination artificielle avec paillettes au profit de la monte classique, moins chère mais pouvant générer des animaux moins performants pour les éleveurs. Enfin, la transformation locale en fromages et yaourts s'est rapidement développée pour compenser l'absence de vente de lait frais et absorber les quantités qui n'étaient plus achetées par TIKO, en particulier pour les années 2009 et 2010 (au détriment de la qualité globale des fromages). La filière a finalement retrouvé ses marques en 2012 avec un accroissement du volume des ventes de lait frais sur Antananarivo par des collecteurs spécialisés.

Au niveau de l'atelier laitier, toutes les exploitations enquêtées ont subi une perte nette en 2009 et 2010 par rapport à 2008, suite au manque de débouchés et à un prix du lait très bas (tableaux V et VI). La situation s'est nettement améliorée en 2011 puis en 2012, avec une augmentation significative du prix du lait (de 600 à $1000 \mathrm{Ar} / \mathrm{L}$, puis 1200 Ar/L début 2014), montrant que l'offre n'avait toujours pas rattrapé la demande qui restait croissante sur Antananarivo, où les élevages laitiers périurbains se sont développés depuis 2011. Mécaniquement, les producteurs du Vakinankaratra ont retrouvé des marges positives du fait de la conjonction d'un prix de vente attractif, de l'amélioration du niveau de production par vache laitière et de la baisse structurelle de leurs charges opérationnelles.

Les impacts de la crise de 2009 ont été plus importants dans la zone de Betafo (tableau VI), où les principaux exploitants touchés ont été ceux possédant un cheptel important (plus de quatre vaches laitières). Ceux-ci ont connu en 2009 une baisse de revenu importante par rapport à l'année 2008. En 2010 à Betafo, la baisse d'activité de l'atelier laitier a été moindre qu'en 2009, mais les exploitants ont gagné en moyenne par exploitation $49 \%$ de moins qu'en 2008 sur la vente du lait. Dans la zone périurbaine de Vinaninkarena, la baisse d'activité de l'atelier laitier a également été importante en 2009 (- $46 \%$ 
Tableau V

Recettes et pertes nettes de l'atelier laitier en fonction des systèmes d'alimentation sur les hautes terres à Madagsacar entre 2008 et 2010

\begin{tabular}{|c|c|c|c|c|c|c|c|c|c|}
\hline \multirow[t]{2}{*}{ Expl } & \multirow[t]{2}{*}{ Sys } & \multirow[t]{2}{*}{ TC } & \multicolumn{3}{|c|}{$\begin{array}{l}\text { Recette de l'élevage } \\
\text { laitier (k.ariary) }\end{array}$} & \multicolumn{2}{|c|}{$\begin{array}{l}\text { Baisse des revenus nets } \\
\text { par rapport à année de } \\
\text { référence } 2008 \text { (k.ariary) }\end{array}$} & \multicolumn{2}{|c|}{$\begin{array}{l}\text { Baisse des revenus nets } \\
\text { par rapport à année de } \\
\text { référence } 2008(\%)\end{array}$} \\
\hline & & & 2008 & 2009 & 2010 & 2009 & 2010 & 2009 & 2010 \\
\hline Ants 2 & $\mathrm{~S} 1$ & A & 1780 & 1400 & 555 & -380 & -1225 & -21 & -68 \\
\hline Ants 4 & $\mathrm{~S} 1$ & B & 2016 & 1314 & 690 & -702 & -1326 & -34 & -65 \\
\hline Vin 3 & S2 & A & 1656 & 702 & 768 & -954 & -888 & -57 & -53 \\
\hline Vin 6 & S2 & B & 1098 & 864 & 1140 & -234 & 42 & -21 & 3 \\
\hline Bet 3 & S4 & $\mathrm{C}$ & 4093 & 1965 & 2538 & -2128 & -1555 & -51 & -37 \\
\hline Bet 5 & S4 & $\mathrm{C}$ & 12312 & 3566 & 5820 & -8746 & -6492 & -71 & -52 \\
\hline
\end{tabular}

Expl : exploitant ; Sys : système d'alimentation ; TC : type de complément Ants : Antsapanimahazo; Vin : Vinaninkarena ; Bet : Betafo

des revenus nets par rapport à 2008). Dans cette zone, le marché est aujourd'hui partiellement restauré mais reste fragile et peu sécurisé : beaucoup de paysans se plaignent de ne pas recevoir l'intégralité de leurs paiements au retour du collecteur. A Antsapanimahazo, la

\section{Tableau VI}

Revenus nets moyens de I'atelier laitier entre 2008 et 2010 selon les zones d'étude ( $n$ 'incluent pas les ventes $d^{\prime}$ animaux pour rester dans le comparatif avant et après la crise) sur les hautes terres à Madagsacar

\begin{tabular}{|c|c|c|c|c|}
\hline & \multicolumn{2}{|c|}{$\begin{array}{l}\text { Baisse des revenus nets } \\
\text { par rapport à l'année } \\
\text { de référence } 2008 \text { (Ar) }\end{array}$} & \multicolumn{2}{|c|}{$\begin{array}{l}\text { Baisse des revenus nets } \\
\text { par rapport à l'année } \\
\text { de référence } 2008(\%)\end{array}$} \\
\hline & 2009 & 2010 & 2009 & 2010 \\
\hline Ants & -451000 & -694000 & -36 & -47 \\
\hline Vin & - 1031000 & -976000 & -46 & -35 \\
\hline Bet & -3250000 & -1050000 & -63 & -49 \\
\hline
\end{tabular}

N.B. : les ventes d'animaux ont servi principalement dans un premier temps à compenser la mévente du lait pendant la première année après la crise et à investir dans des cycles de production animale plus courts (porc).

Ants : Antsapanimahazo ; Vin : Vinaninkarena ; Bet : Betafo baisse des revenus laitiers en 2009 a été plus faible que dans les autres zones. En 2010, la baisse s'est accrue avec $47 \%$ de revenus nets de moins qu'en 2008 sur l'atelier laitier. Ceci était dû à la forte diminution de l'alimentation des vaches laitières, qui a eu pour conséquence de diminuer drastiquement leur production, ainsi qu'à la difficulté encore importante de trouver des débouchés pour la vente de lait fin 2009. Le marché du lait a depuis 2010 fortement augmenté, d'où une augmentation des prix.

\section{Conséquences de l'arrêt de la distribution d'une provende commerciale de qualité}

Les exploitants qui alimentaient leur cheptel avec de la provende commerciale (produite par la société TIKO en particulier) ont dû changer de complément alimentaire avec la disparition des distributeurs après la crise de 2009. Plusieurs cas ont ainsi été observés : a) les éleveurs qui ont fabriqué une provende à faible valeur nutritive de type $\mathrm{A}$; b) les éleveurs qui ont distribué une provende de qualité moyenne de type $\mathrm{B}$, souvent à base d'excédents vivriers issus de l'exploitation (grains de maïs et de soja, son de riz); et c) les éleveurs qui ont voulu maintenir une production laitière suffisante et ont suivi les formules préconisées par les techniciens associant des composés plus énergétiques et protéiques (notamment farine de maïs + tourteau) de type $\mathrm{C}$.

Le tableau VII montre les exploitants ayant changé la provende commerciale pour une provende de type A (de moindre qualité) et une diminution de la production laitière annuelle de l'ordre en moyenne

\section{Tableau VII}

Dépenses et rentabilité des exploitants laitiers distribuant une provende à faible valeur alimentaire sur les hautes terres à Madagsacar entre 2008 et 2010

\begin{tabular}{|c|c|c|c|c|c|c|c|c|c|c|c|c|c|c|}
\hline \multirow[t]{2}{*}{ Expl } & \multirow[t]{2}{*}{ Sys } & \multirow[t]{2}{*}{ TC } & \multicolumn{3}{|c|}{$\begin{array}{l}\text { Dép compléments } \\
\text { (k.Ar/an) }\end{array}$} & \multicolumn{3}{|c|}{$\begin{array}{l}\text { Production lait } \\
\text { totale (L/an) }\end{array}$} & \multicolumn{3}{|c|}{$\begin{array}{l}\text { Marge brute de } \\
\text { l'élevage (k.Ar/an) }\end{array}$} & \multicolumn{3}{|c|}{$\begin{array}{l}\text { Coût production } \\
\text { pour } 1 \text { L de lait (Ar) }\end{array}$} \\
\hline & & & 2008 & 2009 & 2010 & 2008 & 2009 & 2010 & 2008 & 2009 & 2010 & 2008 & 2009 & 2010 \\
\hline Ants 1 & S1 & A & 51 & 41 & 41 & 735 & 735 & 740 & 94 & 50 & 81 & 411 & 377 & 310 \\
\hline Ants 2 & S1 & A & 354 & 354 & 265 & 3560 & 3400 & 2160 & 1157 & 777 & 208 & 216 & 183 & 173 \\
\hline Vin 1 & S1 & A & 252 & 184 & 152 & 1500 & 800 & 1000 & 342 & 107 & 339 & 452 & 466 & 361 \\
\hline Vin 3 & S2 & A & 942 & 105 & 105 & 2760 & 1720 & 1680 & 435 & 355 & 451 & 442 & 201 & 188 \\
\hline Vin 2 & S1 & A & 852 & 143 & 143 & 2400 & 1020 & 1100 & 212 & 64 & 74 & 511 & 357 & 330 \\
\hline
\end{tabular}

Expl : exploitant ; Sys : système d'alimentation ; TC : type de complément ; Dép compléments : dépenses en compléments

Ants : Antsapanimahazo ; Vin : Vinaninkarena 
de $30 \%$ sur les six premiers mois de la crise. Les exploitants ayant remplacé la provende commerciale par un concentré à faible valeur nutritionnelle (type A) ont subi une diminution importante de la production laitière et des charges opérationnelles. Le coût de production du lait a été amélioré mais la marge sur l'élevage laitier a été plus faible du fait des difficultés de vente et du faible prix du lait. Notons que la diminution de la production laitière de l'exploitant Ants 1 a été moins importante car il n'achetait pas de provende commerciale en 2008. Il n'a pas changé de formule de provende entre 2008 et 2010 (provende de type A), seules les quantités ont diminué. La fabrication de provende fermière avec les produits de l'exploitation a permis de diminuer fortement les charges opérationnelles. Peu d'exploitants (15\% de l'échantillon) ont diminué en parallèle la ration fourragère ou la surface des cultures fourragères.

\section{Typologie de comportement des éleveurs}

Les exploitants ont réagi de façons différenciées à la crise et avec des objectifs bien spécifiques : minimiser les pertes, s'adapter au marché ou encore trouver de nouveaux débouchés. Sur la base de ces enquêtes nous avons pu définir une typologie des comportements :

- 6 exploitants sur 45 enquêtés (13\%) n'ont pas changé leurs pratiques ; la baisse temporaire du prix du lait n'a pas induit de modification des pratiques chez les exploitants dont l'activité laitière était marginale avant la crise. Ceux-ci n'alimentaient pas leurs animaux avec des provendes achetées et vendaient leur lait sur le marché local. L'impact économique observé chez ces exploitants a été uniquement dû à la baisse momentanée du prix du lait et à la saturation du marché local en 2009 ;

- 39 exploitants $(87 \%)$ ont réagi en modifiant la composition de la provende, en réduisant temporairement leur production de lait, en vendant partiellement leur cheptel. Ils ont également cherché de nouveaux débouchés par différents moyens comme des déplacements quotidiens pour vendre leur lait aux gargotes de la ville, l'organisation de navettes journalières en taxi-brousse afin d'acheminer le lait sur le marché d'Antsirabe et l'émergence de nouveaux collecteurs privés (des producteurs locaux). Trois de ces 39 éleveurs se sont adaptés par la diversification (porc) et la transformation (fromage/yaourt).

Après une phase d'adaptation et de réorganisation, la filière a globalement retrouvé courant 2012 un dynamisme important, avec une

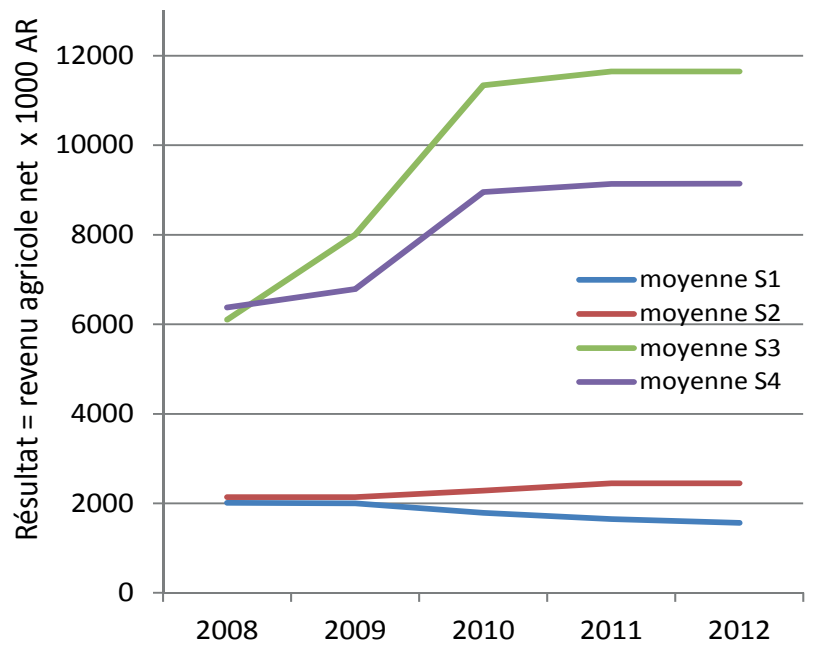

Figure 8 : évolution des résultats (revenu agricole net), issus du compte d'exploitation général par type d'exploitation laitière (de S1 à $S 4$; $S 3$ est composé d'une seule personne) dans la province du Vakinankaratra à Madagascar entre 2008 et 2012. Note : les coefficients de variation de la moyenne varient entre 32 et $43 \%$ pour S1, entre 71 et $90 \%$ pour S2, et entre 37 et $67 \%$ pour $S 4$. production au moins aussi importante qu'en 2008 avant la crise grâce au développement d'une très forte demande sur Antananarivo. L'offre a peiné à satisfaire cette demande avec pour impact le prix du lait coûtant $1000 \mathrm{Ar} / \mathrm{L}$ en 2011 et $1200 \mathrm{Ar} / \mathrm{L}$ en 2013. La plupart des éleveurs qui avaient vendu des vaches en 2009 en ont racheté à partir de 2011. En revanche, la crise les a renforcés, avec une structure du système d'alimentation des animaux plus diversifiée et surtout une source de provende moins chère bien que, dans beaucoup de cas, plus pauvre du point de vue nutritif, ou déséquilibrée dans son ratio énergie/protéine, mais non dépendante d'une société privée majoritaire sur le marché comme cela était le cas avant 2009. La dépendance diminuée des éleveurs vis-à-vis des sociétés privées pour la provende, la baisse des coûts de cette provende, probablement une meilleure connaissance des besoins alimentaires des animaux et la diversification de la production à travers la transformation partielle du lait ont renforcé les exploitations agricoles et les ont rendues globalement plus résilientes au sens de Gunderson et Holling (2002). Cependant, le retour à la monte naturelle et la perte ou l'érosion génétique due à l'arrêt des inséminations artificielles avec des semences de qualité auront sans aucun doute dans les cinq prochaines années un impact négatif sur les performances des ateliers et la production globale de lait (sans impact sur la résistance des animaux aux maladies locales).

La figure 8 montre trois groupes d'exploitations concernant le revenu agricole net en 2010 (résultat issu du CEG) : a) un groupe autour de 2,5 millions d'ariary/an, très fragile, généralement avec une vache laitière et qui a potentiellement souffert de la crise, b) un groupe autour de 5 millions d'ariary/an qui a dû nécessairement s'adapter à la crise et a pu maintenir son revenu, et c) un groupe de cinq éleveurs au-dessus de 7,5 millions d'ariary/an qui, après adaptation, a globalement amélioré son revenu (enquêtes 2 et 3).

On retrouve dans l'analyse de l'évolution des soldes de revenu - correspondant à la capacité réelle d'investissement de l'exploitation en fin d'année utilisée soit dans l'investissement agricole ou non-agricole, soit dans l'amélioration des conditions de vie du ménage - les mêmes trois groupes (figure 9) : a) un groupe qui s'est maintenu difficilement dans l'activité mais a réussi à surmonter la crise à l'exception de trois éleveurs, b) un groupe moyen résilient qui a retrouvé un solde similaire à 2008 après la crise, et c) un groupe de quatre éleveurs aux résultats variés, dont deux ont augmenté leur solde et sont sortis clairement renforcés de la crise, et deux autres ont récupéré en 2012 le même solde qu'en 2008 et sont donc revenus à la situation antérieure, d'où une certaine résilience (enquêtes 2 et 3 ).

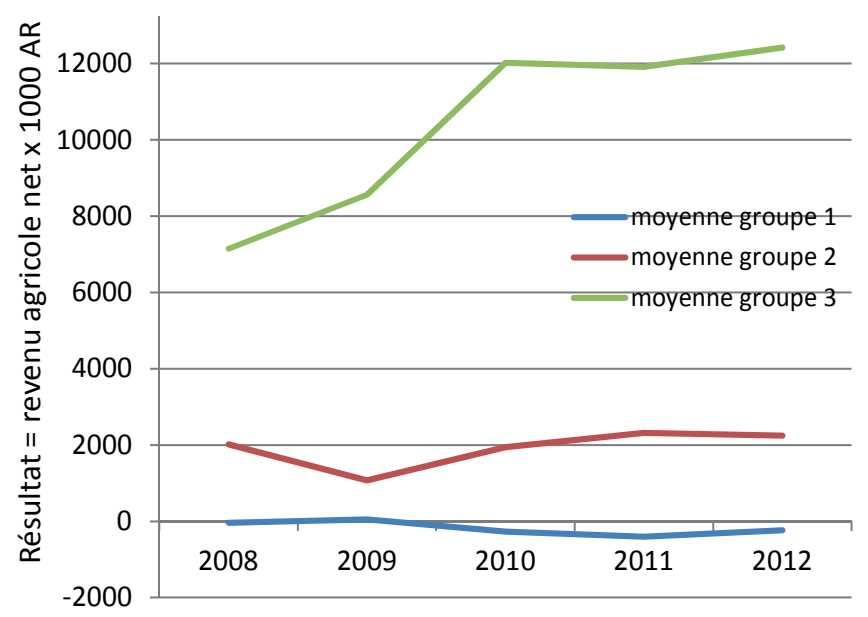

Figure 9 : évolution des soldes de trésorerie (assimilables à la capacité potentielle d'investissement) par groupe d'exploitations laitières dans la province du Vakinankaratra à Madagascar entre 2008 et 2012. 


\section{La situation malgache est elle spécifique ou similaire aux tendances relevées en Afrique?}

En Afrique de l'Ouest et du Centre, la priorité en termes d'intégration agriculture-élevage a été donnée à la mécanisation avec la traction attelée et, dans quelques situations, le ranching pour la production de viande (Dugué et al., 2004). Cependant, les états ouest-africains doivent aujourd'hui faire face à l'explosion de la demande urbaine en lait et importent massivement de la poudre de lait (Corniaux et al., 2012). Pour réduire leur dépendance alimentaire, les gouvernements cherchent alors à développer le secteur laitier. Une multitude de projets de développement sont apparus depuis les années 1970 (Vatin, 1996), des minilaiteries gérées par des coopératives ou des opérateurs privés ont émergé au début des années 2000 au Sénégal, au Mali, au Burkina Faso et au Niger.

Une typologie des exploitations laitières a été réalisée dans trois pays, au Sénégal, en Mauritanie et au Mali, sur la base de 307 exploitations enquêtées entre 1999 et 2006 avec des critères basées sur les structures de production, les performances laitières et les orientations productives (Corniaux et al., 2012). A l'exception d'une poignée de fermes périurbaines spécialisées (Duteurtre, 2007), très peu d'exploitations font du lait le pivot économique de leur système de production, en général fort diversifié. Corniaux et al. (2012) écrivent : « ... stratégie dominante est une stratégie sécuritaire, dans le cadre d'exploitations familiales regroupant plusieurs ménages relativement pauvres qui privilégient la production de céréales ou d'animaux sur pieds ». Mais diversification ne s'oppose pas à intensification de la production laitière. Ainsi au Sénégal, Sow et al. (2007) sur 96 éleveurs, 50 commerçants en produits laitiers et 120 consommateurs montrent que « les modes de production animale tendaient vers l'intensification, avec une réduction des effectifs du fait de la restriction de l'espace pastoral » et un recours plus important aux résidus de culture et aux aliments achetés que par le passé.

Nous retrouvons dans le triangle laitier à Madagascar cette tendance forte à l'intensification avec en général de petits effectifs d'animaux (inférieurs à cinq) mais un développement important des cultures fourragères comme moyen majeur de l'intensification, ce que l'on ne constate pas encore en Afrique de l'Ouest. Le cas malgache est donc original au sens d'une spécialisation des exploitations sur le laitier. La situation d'après crise montre également une très forte résilience sur cette activité laitière, ce qui renforce localement les stratégies d'intensification, de diversification et de spécialisation (monétarisation au détriment de la sécurité alimentaire par l'autoconsommation du vivrier produit).

Les dynamiques de développement du secteur laitier ouest-africain sont donc assez proches de celles de Madagascar, basées sur une demande croissante en lait et produits laitiers dans les villes, même si les contextes sont différents (à l'exclusion de la culture fourragère) et l'intérêt des complémentarités agriculture-élevage, essentielles pour les pays tropicaux (énergie, transfert de fertilité et diversification des revenus), réapparaît dans l'agenda de la recherche et du développement de régions à climat tempéré comme l'Europe (Dugué et al., 2013). La production laitière est une activité complémentaire pour améliorer le revenu total et la trésorerie de l'exploitation, valoriser les résidus de récoltes et fournir une fumure organique à faible coût (souvent la seule source de fumure disponible dans les fermes). Dans le triangle laitier à Madagascar, malgré un foncier cultivable sursaturé et des exploitations très petites centrées sur la satisfaction en priorité de la sécurité alimentaire, le passage à la production laitière est généralement considéré comme un levier pour entrer dans le cercle vertueux du développement. Notre étude dans trois zones du triangle laitier malgache montre aussi que l'intensification de la production laitière, quand elle est raisonnée dans un système de polyculture élevage diversifié, ne constitue pas une prise de risque pour le producteur dans la mesure où il est capable de s'adapter rapidement aux évolutions de son environnement économique.

Dans d'autres pays (Inde, Brésil et Maroc par exemple) les producteurs laitiers bénéficient d'infrastructures (routes, électricité, systèmes de tank à froid pour la collecte, laiteries, et autres), de systèmes d'appui-conseil et de crédit, d'un environnement industriel fournissant les intrants (par exemple aliments, produits vétérinaires) et surtout d'un réel marché solvable en croissance, ce qui manque dans plusieurs pays du continent africain, alors qu'à Madagascar le marché est en pleine expansion. Dans ces pays émergents, l'ensemble des services et infrastructures appuyant la filière laitière a permis aux éleveurs d'accroître rapidement les performances de leurs élevages et de concurrencer ainsi les importations de lait en poudre des pays développés (ou éventuellement de bénéficier d'une politique de régulation et de protection des marchés intérieurs). Cela s'est cependant parfois accompagné d'une spécialisation des exploitations et d'une dépendance vis-à-vis du crédit, des intrants (parfois importés) et surtout des agro-industriels qui tendent à tirer les prix vers le bas (Sraïri et al., 2007), sauf en Inde où la production laitière est principalement issue d'exploitations paysannes mixtes polyculture-élevage.

Les stratégies agricoles des producteurs des pays sahéliens sont centrées sur l'autoconsommation et la sécurité alimentaire, et elles se caractérisent par une faible monétarisation de leur système de production et donc une meilleure durabilité face aux crises. Les produits piliers des systèmes ruraux sahéliens sont les céréales et la viande, comme le sont le riz et le zébu pour Madagascar. Pour le lait, l'Afrique de l'Ouest semble moins bien armée pour relever le défi de l'autonomie alimentaire alors qu'au contraire Madagascar peut intensifier la production dans la zone traditionnelle du triangle laitier mais aussi développer son offre dans les zones proches des grandes villes (la capitale en particulier) et dans la zone du moyen ouest, zone d'émigration à forte vocation agricole et potentiellement d'élevage. Si la demande globale de Madagascar est en hausse, elle reste cependant limitée par un pouvoir d'achat restreint de la population. Le secteur productif a montré une remarquable adaptation à la crise et une aptitude certaine à l'innovation et à répondre à la demande. Le lait frais et ses dérivés ont un bel avenir dans l'île rouge.

La principale différence entre le cas du triangle laitier malgache et celui de l'Afrique de l'Ouest est culturelle et technique. Le lait en Afrique de l'Ouest est le fait d'éleveurs peuls traditionnels qui développent un élevage sur des ressources naturelles gratuites (parcours et vaine pâture). Ils ont tardivement commencé à intensifier. Il y a peu de cultures fourragères, même dans la zone ou le lait est collecté ; les éleveurs préfèrent dépendre de provende achetée ou ne pas ou peu intensifier. Les cultures fourragères sont bien plus présentes à Madagascar sur les hautes terres ou les éleveurs sont tous agriculteurs. La culture fourragère leur permet de gagner du temps et d'accroître notablement leurs capacités d'affouragement ce qui a clairement été montré par Andriarimalala et al. (2013). La situation de Madagascar est en cela plus proche de celle de l'Afrique de l'Est (hauts plateaux et climat moins aride permettant la production fourragère, et des conditions de vie plus favorables pour les animaux au regard de la température par exemple).

\section{CONCLUSION}

Deux situations de production laitière sont apparues nettement dans le triangle laitier malgache. Dans la première se trouvaient les petites et moyennes exploitations laitières des hautes terres du Vakinankaratra, très hétérogènes tant au niveau technique (niveau d'intensification) que structurel (niveau de diversification des activités) ; la plupart disposait de revenus diversifiés, associant l'agriculture et l'élevage, constituant un élément de forte capacité d'évolution et une 
certaine flexibilité dans l'adaptation, avec la baisse du prix du lait et la réduction importante des quantités commercialisées, suite à la crise politique de 2009 et à la disparition du groupe TIKO. Dans la deuxième les exploitants ayant intensifié leur production de lait avant 2009 et dont les revenus étaient essentiellement basés sur la vente du lait ont dû s'adapter plus radicalement à cette crise et ont pris le risque de changer leur système d'exploitation en diminuant le nombre de vaches laitières ou en réduisant leur coût de production en fabricant eux-mêmes la provende dont la qualité était variable. Dans certains cas le niveau d'intensification de leur élevage a diminué (provende de faible qualité nutritionnelle et arrêt de l'insémination artificielle).

Pour les petites exploitations laitières, l'amélioration de la ration alimentaire à court terme (complément alimentaire) et le niveau de l'amélioration du capital génétique des troupeaux sur le long terme sont les deux points clés de la rentabilité et de la durabilité des élevages. Dans les exploitations les plus intensifiées, les enjeux résident actuellement dans le maintien d'une activité laitière importante, tout en assurant une meilleure résilience globale aux chocs potentiels par la diversification des sources de provende et l'amélioration à moindre coût des rations (valorisation optimale des résidus et des sous-produits pour l'élevage de l'exploitation). Ces exploitations gagnent en autonomie si elles fabriquent des provendes fermières de bonne qualité, tout en développant d'autres activités (notamment élevage de porcs, autres élevages, fromages) afin de minimiser les risques en cas de nouvelle chute du prix du lait. L'intégration agriculture-élevage est un point clé du développement du Vakinankaratra. Nous avons montré l'importance de la valorisation des produits agricoles de l'exploitation dans l'alimentation des vaches laitières afin de ne plus dépendre des provendes du commerce et pour la fertilisation animale qui est bien souvent le seul engrais (organique) utilisé sur les hautes terres en l'absence de fertilisation minérale.

Les exploitations laitières du triangle laitier sortent renforcées de cette crise avec des modes de production plus souples et mieux adaptés à des crises potentielles futures sur les prix (système moins intégré que celui prôné par la société TIKO avant la crise). Toutefois l'offre de lait ne satisfaisait pas encore la demande en 2014 qui avait fortement augmenté depuis 2010, notamment en raison de l'augmentation démographique, ce qui assure pour les prochaines années un débouché garanti pour les producteurs locaux. La crise a ainsi permis de renforcer la résilience des exploitations et la résilience globale de la filière (nouveaux opérateurs et transformateurs). Il est paradoxal de voir que finalement cette crise a renforcé un milieu agricole pourtant réputé fragile et particulièrement intensif sur les hautes terres, montrant ainsi un exemple de possibilité d'adaptation et de souplesse d'une petite agriculture familiale à des changements structurels de la filière importants.

Cependant, depuis 2009, il y a eu une forte réduction du conseil technique, de la fourniture de paillettes d'insémination artificielle (très importante sur le long terme) suite à la baisse d'activité de Fifamanor (et l'arrêt du projet BVPI-SE/HP en novembre 2012). Sur le long terme, cela pourrait avoir un impact négatif sur le potentiel de production des vaches laitières comme sur la sensibilisation aux pratiques d'alimentation et d'hygiène. Il serait souhaitable de renforcer le rôle déjà essentiel des techniciens locaux des organisations paysannes, coopératives et autres structures pérennes, afin d'assurer une plus large diffusion des informations techniques sur le rationnement des animaux, la composition des provendes et l'utilisation des pâturages, avec un appui plus poussé en termes de conseils techniques.

Il apparaît également nécessaire de réaliser une estimation de l'évolution à terme du marché national pour mieux adapter l'offre à la demande et enregistrer les tendances et trajectoires. Par exemple le développement récent de la production laitière autour d'Antananarivo pourrait à terme limiter le déplacement du triangle laitier actuel vers la capitale et avoir un impact sur les prix. Madagascar importe également du lait en poudre pour les gros industriels (Socolait) qui pourrait aussi concurrencer la production locale selon le type de politique appliqué a ces importations.

Alors que les anciennes structures de TIKO ont partiellement redémarré en 2012-2013 et que la société Socolait a augmenté la collecte de lait frais sur la région, il serait intéressant d'évaluer à terme les conséquences (au niveau du marché, de l'évolution sur les prix) de la réémergence de ces opérateurs privés de poids et d'observer la robustesse des petites filières locales (fromages et yaourts, gargotiers, par exemple) qui se sont développées ces deux dernières années pour compenser la perte d'un acheteur qui représentait $50 \%$ du lait produit. Il faudrait ultérieurement observer à moyen terme la trajectoire des exploitants face à l'évolution politique du pays (qui détermine en grande partie l'activité économique globale du pays) et déterminer les conséquences à long terme des adaptations effectuées lors de cette crise de 2009. Enfin, il serait important de savoir si la filière laitière peut se développer sur la base d'un marché local ou national (non encore saturé mais avec un pouvoir d'achat globalement en baisse) et d'en définir les acteurs, ou bien si la présence de gros industriels comme TIKO ou Socolait reste une nécessité pour relancer un secteur resté globalement artisanal.

\section{REFERENCES}

Ahmim-Richard A., Bodoy A., Penot E., Rahaison A., 2011. Modélisation des exploitations agricoles comme outil d'aide à la décision pour les projets des zones Hauts Plateaux et Moyen Ouest de Madagascar. In: Exploitations agricoles, stratégies paysannes et politiques publiques : les apports du modèle Olympe. Quae, Versailles, France, 131-146

Andriamirija M.H., 2009. Analyse des facteurs déterminants de la transformation laitière dans la région du Vakinankaratra. DEA Agromanagement, ESSA, Université d'Antananarivo, Madagascar, 50 p.

Andriarimalala J., Rakotozandriny J.D.N., Andriamandroso A.L.H., Penot E., Naudin K., Dugué P., Tillard E., Decruyenaere V., Salgado P., 2013. Creating synergies between conservation agriculture and cattle production in crop-livestock farms: A case study in the lake Alaotra region of Madagascar. Exp. Agric., 49 (3): 352-365, doi : 10.1017/ S0014479713000112

Carimentrand A., 2011. Le lait : une filière en recomposition après la suspension des activités du groupe TIKO. In : Madagascar dans la tourmente (éds Andrianirina N., Ballet J., Nirina Rabevohitra B., Rasofolo P.). L'Harmattan, Paris, France, 79-87

Corniaux C., Alary V., Gautier D., Duteurtre H., 2012. Producteur laitier en Afrique de l'Ouest : une modernité rêvée par les techniciens à l'épreuve du terrain. Autrepart, 3 (62) : 17-36, doi : 10.3917/ autr.062.0017

Duba G., 2010. Modélisation des exploitations agricoles intégrant une forte proportion d'activités d'élevage. Mém. Master, Epsed, université de Montpellier, France, 80 p.

Dugué P., Andrieu N., Blanchard M., Havard M., Le Gal P.-Y., Penot E., Salgado P., Vall E., 2013. Les relations agriculture élevage en Afrique subsaharienne : quelques enseignements pour l'agriculture européenne. Séminaire Agriculture de demain et enjeux d'aujourd'hui, Toulouse, France, 4 juin 2013, 20 p.

Dugué P., Vall E., Lecomte P., Klein H.D., Rollin D., 2004. Evolution des relations entre I'agriculture et l'élevage dans les savanes d'Afrique de l'Ouest et du Centre. Un nouveau cadre d'analyse pour améliorer les modes d'intervention et favoriser les processus d'innovation. OCL, 11 : $(4 / 5): 268-276$

Duteurtre G., 2007. Trade and development of dairy production in West Africa: a review [in French]. Rev. Elev. Med. Vet. Pays Trop., 60 (1-4): 209-223

Fifamanor, 2008. Rapport annuel 2007. Fifamanor, Antsirabe, Madagascar, $83 \mathrm{p}$.

Fifamanor, TAFA, GSDM, Cirad, URP, Sicalait, 2008. Conduite des systèmes de culture sur couverts végétaux et affouragement des vaches laitières. Guide pour les Hautes Terres de Madagascar. ARP, La Réunion, France, 92 p. 
Gunderson L.H., Holling C.S., Eds, 2002. Panarchy: understanding transformations in human and natural systems. Island Press, Washington DC, USA, 360 p.

Kasprzyk M., Penot E., Dugué P., 2008. Diversité des systèmes d'alimentation des troupeaux bovins laitiers à Betafo, région du Vakinankaratra, Madagascar. AFD, Paris, France, 38 p. (Coll. AFD/ Sehmpo BV lac $n^{\circ} 10$

Penot E., 2012. Exploitations agricoles, stratégies paysannes et politiques publiques. Les apports du modèle Olympe. Quae, Versailles, France, 350 p. (Coll. Update Sciences \& Technology)

Penot E., Razanakoto Niriharisoanjanahary M., 2012. Etude des circuits de commercialisation du lait et de ses dérivés dans la région du Vakinankaratra en 2011. Corus/EU/Cirad, Antananarivo, Madagascar, 45 p. (Coll. AFD/BVPI-SE/HP Document de travail $n^{\circ} 43$ )

Rabefenomanantsoa A., 2009. Facteurs déterminants de la consommation et de la commercialisation du lait et des produits laitiers dans la région de Vakinakaratra. Cas d'Antsirabe I, d'Antsirabe II, et de Betafo. DEA Agro-management, ESSA, Université d'Antananarivo, Madagascar, 58 p.

Rabemanambola M., Rakotoarisoa J., Rieutort J., 2009. Entre ville et campagne : les adaptations du maraîchage paysan sur les Hautes Terres centrales malgaches. Cah. Outre-Mer, (247) : 285-310
Randrianasolo J., Penot E., 2008. Analyse des exploitations laitières sur les hautes terres. Cirad, Montpellier, France CD-rom (Coll. documents de travail à Madagascar $\left.n^{\circ} 4\right)$

Rarivoarimanana B., 2010. Analyse des systèmes d'alimentation des bovins laitiers à Vinanikarena et à Antsapanimahazo, région du Vakinankaratra, Madagascar. Master Agronomie spécialisation élevage, ESSA, Université d'Antananarivo, Madagascar, 65 p.

Rarivoarimanan, Penot E., Rabearimisa R., 2010. Diversité des systèmes d'alimentation des troupeaux laitiers à Vinaninkarena et à Antsampanimahazo Faratsiho, région Vakinankaratra. Ministère de I'Agriculture, de l'Elevage et de la Pêche, Antananarivo, Madagsacar. (Coll. BVPI/Scrid/Fofifa/TAFA Doc. Travail $n^{\circ} 41$ )

Sow Dia F., Somda J., Kamuanga M., 2007. Dynamics of the dairy subsector in the Sahelian area: Milk supply and demand in the central agropastoral zone of Senegal [in French]. Rev. Elev. Med. Vet. Pays Trop., 60 (1-4): 77-88

Sraïri M.T., Ben Salem M., Bourbouze A., Elloumi M., Faye B., Madani T., Yakhlef H., 2007. Analyse comparée de la dynamique de la production laitière dans les pays du Maghreb. Cah. Agric., 16 (4) : 251-257, doi : 10.1684/agr.2007.0118

Vatin F., 1996. Le lait et la raison marchande. Essais de sociologie économique. Presses universitaires de Rennes, France, 205 p.

\section{Summary}

Penot E., Duba G., Salgado P., Dugué P. Adaptability of dairy farms in the highlands of the Vakinankaratra province of Madagascar: Impacts of the 2009 crisis

The objective of the study was to understand the practices of Malagasy dairy farmers after the 2009 economic (and political) crisis, and to assess its impact on farm incomes. The analysis focused on the coping strategies of dairy farmers to maintain the income of their farms in the face of this crisis. It was based on surveys conducted between 2008 and 2010. The data covered three areas representing the diversity of the dairy triangle in the highlands, main milk producing region of Madagascar. A typology was firstly carried out on a sample of 59 farms to understand the rationality of farming practices, in particular animal feeding. Secondly, the economic modeling of 21 of them was performed with Olympe software to estimate the impact of the crisis on the income of dairy farmers. Prospective scenarios reflecting the efficiency and risk of technical choices by the farmers served to explore the evolution of the situation before and after the crisis, and to show some resilience of farms.

Keywords: dairy cattle, economic crisis, impact assessment, animal feeding, resilience, Madagascar

\section{Resumen}

Penot E., Duba G., Salgado P., Dugué P. Capacidad de adaptación de las explotaciones lecheras de las tierras altas de la provincia Vakinankaratra en Madagascar: impacto de la crisis de 2009

El objetivo del estudio era el de comprender la racionalidad de las prácticas de los productores de leche de Madagascar después de la crisis económica (y política) de 2009, así como la evaluación de su impacto sobre los ingresos agrícolas. El análisis se centro en las estrategias de adaptación de los productores de leche para mantener los ingresos agrícolas frente a esta crisis. Se basan en encuestas realizadas entre 2008 y 2010. Los datos cubren tres áreas, que representan la diversidad de las tierras altas del triángulo lechero, principal zona productora de leche de Madagascar. Primeramente se llevó a cabo una tipología en una muestra de 59 granjas, con el fin de entender la racionalidad de las prácticas agrícolas, especialmente la alimentación animal. En segundo lugar, una modelización económica con el programa Olympe se apoyó sobre 21 de estas para estimar el impacto de la crisis sobre los ingresos de los productores lecheros. Posibles escenarios prospectivos, mostrando la eficiencia y los riesgos de las escogencias técnicas por los productores, permiten explorar la evolución de la situación antes y después de la crisis, y mostrar una cierta resistencia de las explotaciones.

Palabras clave: ganado lechero, crisis económica, evaluación del impacto, alimentación de los animales, resiliencia frente a una crisis, Madagascar 
